\title{
Selectively implanted subcollector DHBTs
}

\author{
N. Parthasarathy, Z. Griffith, C. Kadow , U. Singisetti, and M.J.W. Rodwell \\ Department of Electrical and Computer Engineering, University of California, Santa Barbara, CA, USA \\ E-mail: navin@ece.ucsb.edu, 805-8933273
}

\author{
M. Urteaga, K. Shinohara, B. Brar \\ Rockwell Scientific Company, Thousand Oaks, CA, USA \\ * now with Infineon Technologies AG, München, Germany
}

\begin{abstract}
$\mathrm{In}_{0.53} \mathrm{Ga}_{0.47} \mathrm{As} / \mathrm{InP}$ double heterojunction bipolar transistors with implanted subcollectors have been designed and fabricated to eliminate the base access pad capacitance. A blanket Fe implant eliminates the interface charge and a patterned $\mathrm{Si}$ implant creates an isolated $N^{++}$subcollector. The extrinsic base-collector capacitance $C_{c b}$ associated with the base interconnect pad $\left(25 \%\right.$ of the total $\left.C_{c b}\right)$ is thus eliminated. These implanted subcollector DHBTs have $363 \mathrm{GHz} f_{t}$ and $410 \mathrm{GHz} f_{\max }$. The DC current gain $\beta \sim 40, B V_{\text {ceo }}=5.6 \mathrm{~V}$, $B V_{c b o}=6.9 \mathrm{~V}\left(I_{C}=1 \mathrm{~mA}\right)$.
\end{abstract}

\section{Introduction}

Development of digital logic and mixed-signal systems operating at higher clock speeds and bandwidth require continued improvement in transistor performance [1]. Projected HBT performance for $160 \mathrm{~Gb} / \mathrm{s}$ systems include an $f_{\tau}$ and $f_{\max }>450 \mathrm{GHz}$, a breakdown voltage $V_{B R, C E O}>3 \mathrm{~V}$, operating current density $J_{e}>10 \mathrm{~mA} / \mu \mathrm{m}^{2}$ at $V_{c b}=0 \mathrm{~V}$, and low base-collector capacitance $\left(C_{c b} / I_{c}<0.5 \mathrm{ps} / \mathrm{V}\right)$ [2]. When designing an HBT for use in a digital IC, it should be done with emphasis on minimizing the major delay terms associated with $\tau=C_{c b} \Delta V_{\text {logic }} / I_{c}$. The base collector capacitance $C_{c b}$, thus contributes significantly to analog bandwidth and digital delay. The parasitic capacitance under the base access pad is non scaleable and is a significant fraction of the total base collector capacitance, especially for shorter length devices used in high speed, low power logic circuits. A popular approach to eliminate this base access pad capacitance is through the use of micro air-bridges [3]. Typically in such processes, the subcollector under the feed lines from the base pad is removed by anisotropic wet etching. The base feed lines therefore are very narrow $\sim 0.6-0.8 \mu \mathrm{m}$ and these thin lines can present a large access resistance, and poses a reliability issue.

Selective ion implantation is a viable alternative to eliminate the base access pad capacitance. Si can be implanted selectively to form the $N^{++}$subcollector. However, the InP growth interface has an N-type charge $\left(10^{12}-10^{13} \mathrm{~cm}^{-2}\right)$ associated with it [4] which is also present under the base access pad. The access pad capacitance is not eliminated unless this charge is depleted or suppressed. Earlier results have used process and growth techniques $[4,5]$, to eliminate this interface charge. Here a shallow Fe implant, a mid gap acceptor in InP, is used to compensate the interface charge. We report here an InP double heterojunction bipolar transistor employing $\mathrm{Fe}$ and selective $\mathrm{Si}$ implant to eliminate the parasitic base collector capacitance associated with the base access pad area, which is $\sim 25 \%$ of the total $C_{c b}$. These devices exhibit $361 \mathrm{GHz} f_{\tau}$ and $404 \mathrm{GHz} f_{\max }$. In addition to the elimination of $C_{c b}$ in the base interconnect pad area, this process provides the following enhancements over existing implanted InP HBT processes: compensation by the $\mathrm{Fe}$ implant of charge at the epitaxial growth interface, a single MBE growth and increased wafer planarity. In the first demonstration of this process, the $f_{\tau}$ and $f_{\max }$ are the highest reported for DHBTs with such implanted subcollectors. Prior to this work, the highest $f_{\tau}$ and $f_{\max }$ reported for InP HBTs with similarly implanted subcollectors are $252 \mathrm{GHz}$ and $283 \mathrm{GHz}$ respectively [6].

\section{Process details}

The process flow of the implanted subcollector HBT with Fe is shown in Fig. 1. A semi-insulating InP substrate is implanted with $\mathrm{Fe}$ at $10 \mathrm{keV}$, at a fluence of $2 \times 10^{13}$ ions- $\mathrm{cm}^{-2}$ (Fig. 1a) and annealed at $700^{\circ} \mathrm{C}$ for 5 minutes. The Fe implant conditions are selected to fully compensate the N-type charge at the growth interface between the substrate and collector epitaxial layers over the observed $1-5 \times 10^{12} \mathrm{~cm}^{-2}$ range of regrowth interface charge densities. The sample is then selectively implanted (Fig. 1b), using $\operatorname{SiN}_{\mathrm{x}}$ as the implant mask, with $\mathrm{Si}$ at $200 \mathrm{keV} / 4 \times 10^{14}$ ions- $\mathrm{cm}^{-2}, 40 \mathrm{keV} / 7 \times 10^{13}$ ions- $\mathrm{cm}^{-2}$ and $10 \mathrm{keV} / 3 \times 10^{13}$ ions- $\mathrm{cm}^{-2}$, and then activated by annealing at $800^{\circ} \mathrm{C}$ for $10 \mathrm{~s}$. The $\mathrm{Si}$ implant dose is much larger then the Fe acceptor density $\left(n_{F e}\right)$ in the selectively implanted region. This ensures that a highly $N^{++}$doped, isolated subcollector region (Fig 1b) is formed. The active InP HBT layers are then grown by MBE. The device $N$ drift collector, base, and emitter layers are identical to that reported in [7]. Growth is initiated with a $3.5 \mathrm{~nm}$ undoped InGaAs etch-stop layer between the InP collector and the InP substrate. The $N$ drift collector, base, and emitter layers are grown, and devices are formed by wet-etching the emitter and base mesas. With the selectively implanted subcollector, HBT isolation does not require a mesa etch, reducing the HBT mesa height and hence improving planarity by $\sim 500 \mathrm{~nm}$. As seen in Fig. 2, the base 
access pad lies outside the subcollector implant boundary and does not contribute to $C_{c b}$.

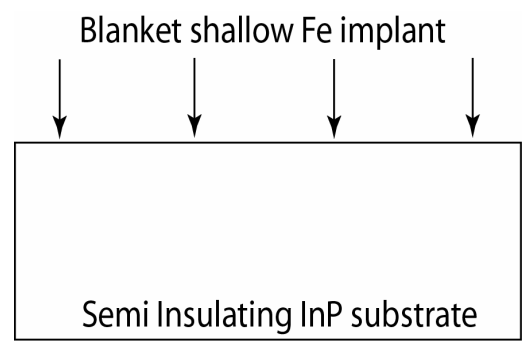

(1a)

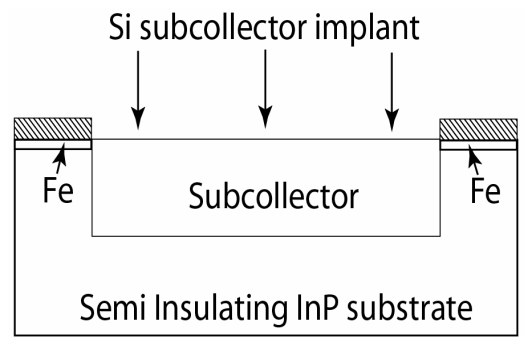

(1b)

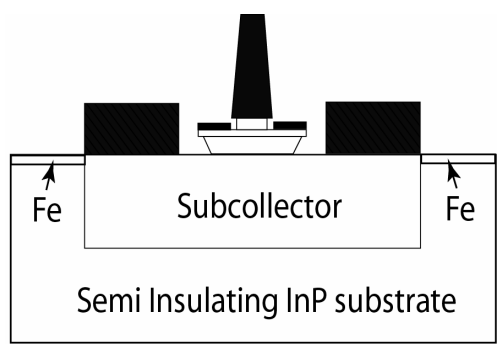

(1c)

Figure 1: Process flow of the implanted subcollector DHBT

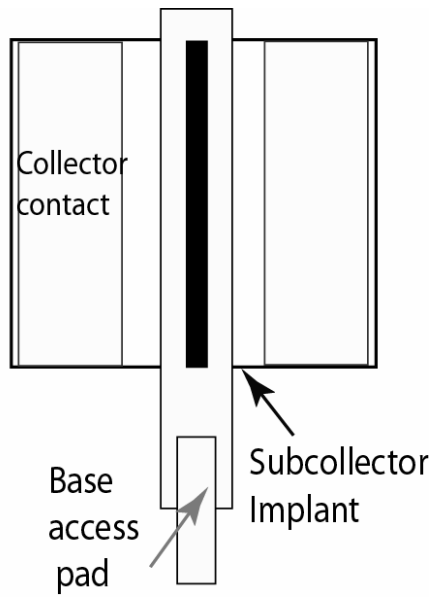

Figure 2: Top view of the Implanted Subcollector DHBT

\section{Measurements and Results}

Hall measurements indicate that the activation of the implanted $\mathrm{Si}$ dopants in the subcollector is $\approx 65 \%$ and the electron mobility is $\approx 900 \mathrm{~cm}^{2} / \mathrm{V}-\mathrm{s}$. AFM scans of the implanted surface after anneal show a mean roughness of $\approx 1$ $\mathrm{nm}$, which is comparable to virgin InP substrate. The isolation between devices is $=10 \mathrm{pA} / \mu \mathrm{m}$. Standard transmission line measurements (TLM) were performed to extract the sheet resistance $\rho_{s}$ and contact resistance $\rho_{c}$ for the base and collector layers. The base sheet resistance $\rho_{s} \approx 1050 \Omega$ and contact resistance, $\rho_{c} \cong 50 \Omega \mu \mathrm{m}^{2}$. The sheet resistance of the implanted subcollector, $\rho_{s} \approx 25 \Omega$. The collector contact $(\mathrm{Ni} / \mathrm{Ge} / \mathrm{Au} / \mathrm{Ni} / \mathrm{Au})$ is made to $\mathrm{InP}$ and partially annealed (320 C / 60s). The anneal temperature was kept low because higher temperature anneals increased the base $\rho_{c}$ on test samples. Consequently, the collector $\rho_{c} \approx 500 \Omega \mu \mathrm{m}^{2}$. From RF parameter extraction, emitter $\rho_{c}$ is $\approx 10 \Omega \mu \mathrm{m}^{2}$. Fig. 3 is a plot of the leakage currents, $I_{C B O}$ and $I_{C E O}$. From this, $V_{C B O}=$ $6.8 \mathrm{~V}$ and $V_{C E O}=5.6 \mathrm{~V}$ at $I_{C}=1 \mathrm{~mA}$.

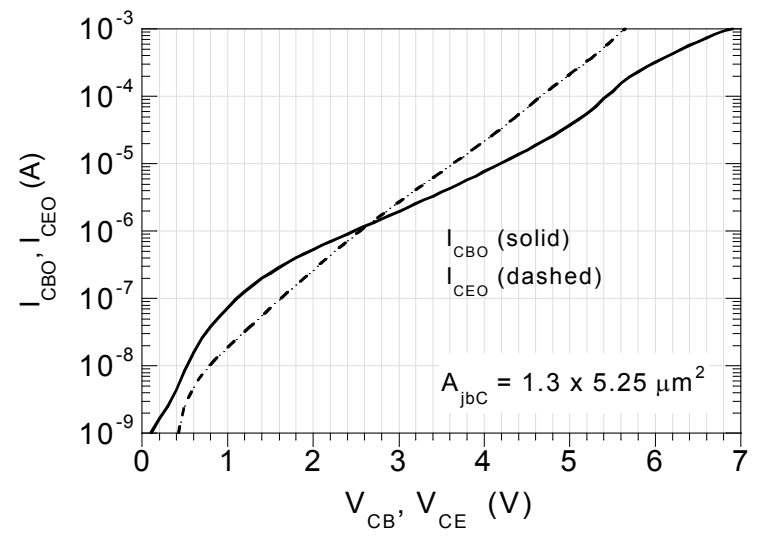

Figure 3: Device leakage currents $I_{C E O}$ and $I_{C B O}$

The DC $I_{C^{-}} V_{C E}$ characteristics are in Fig. 4 and the Gummels in Fig. 5. The maximum DC current gain $\beta$ is 40 . The collector and base ideality factors are, $\eta_{c}=1.18$ and $\eta_{b}=1.58$. These, and the device leakage currents are consistent with those measured for the equivalent triple mesa DHBT [7].

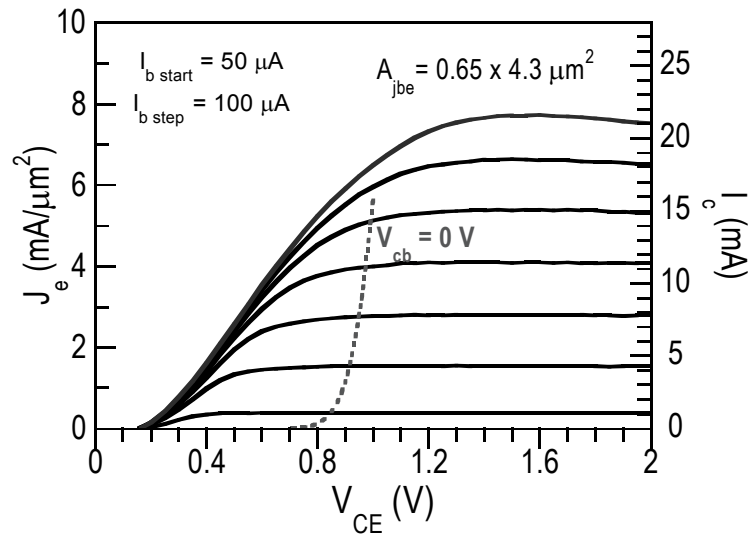

Figure 4: $D C I_{C}-V_{C E}$ characteristics 


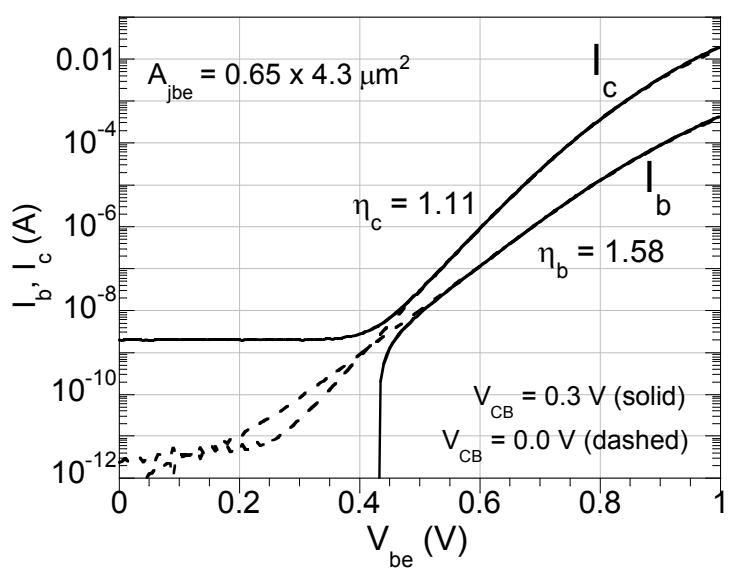

Figure 5: DC Gummel characteristics at $V_{C B}=0 \mathrm{~V}$ and $0.3 \mathrm{~V}$

DC-45 GHz RF measurements were carried out after performing an off wafer Line-Reflect-Reflect-Match (LRRM) calibration on an Agilent $8510 \mathrm{C}$ network analyzer. An onwafer open circuit pad structure identical to the one used by the devices was measured after calibration to de-embed the associated parasitics from the device measurements. Peak $f_{\tau}$ and $f_{\max }$ were determined from extrapolation through a leastsquare-fit to the measured microwave gains $\left|\mathrm{h}_{21}\right|$ and $|\mathrm{U}|$, at measured frequencies. This DHBT has a $0.65 \times 4.3 \mu^{2}$ emitter s/c junction area $A_{j b e}$ and $1.3 \mu \mathrm{m}$ collector mesa width.

These first generation implanted subcollector DHBTs have a maximum $361 \mathrm{GHz} f_{\tau}$ and $404 \mathrm{GHz} f_{\max }$ (Fig. 6) at $I_{c}=17 \mathrm{~mA}$ and $V_{c e}=1.96 \mathrm{~V}\left(J_{e}=6.1 \mathrm{~mA} / \mu \mathrm{m}^{2}\right)$. The $f_{\tau}$ and $f_{\max }$ are lower than the standard mesa-DHBT [7]. We attribute this to the higher base sheet resistance and very high collector series resistance due to the poor ohmic contacts to $N^{++} \mathrm{InP}$.

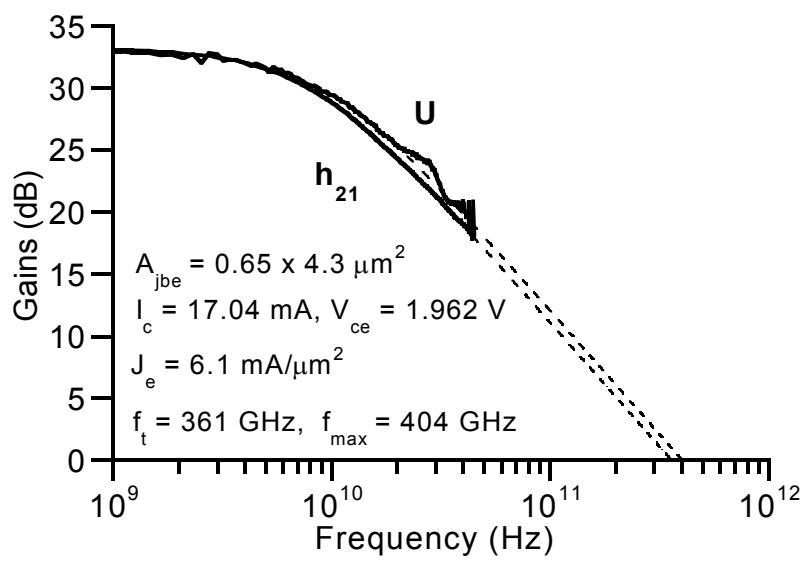

Figure 6: Measured microwave gains

Fig. 5 compares the variation of the total $C_{c b}$ with $V_{c b}$ between the selectively implanted subcollector DHBT and a triple mesa DHBT, both with similar $120 \mathrm{~nm}$ collectors and device dimensions identical [7]. The compensation of interface charge is evident, as seen by the reduction in $C_{c b}$ over the entire measured range of bias voltages. For the implanted subcollector DHBTs, $C_{c b}$ is reduced by $\sim 25 \%$ corresponding to the elimination of the base access pad capacitance.

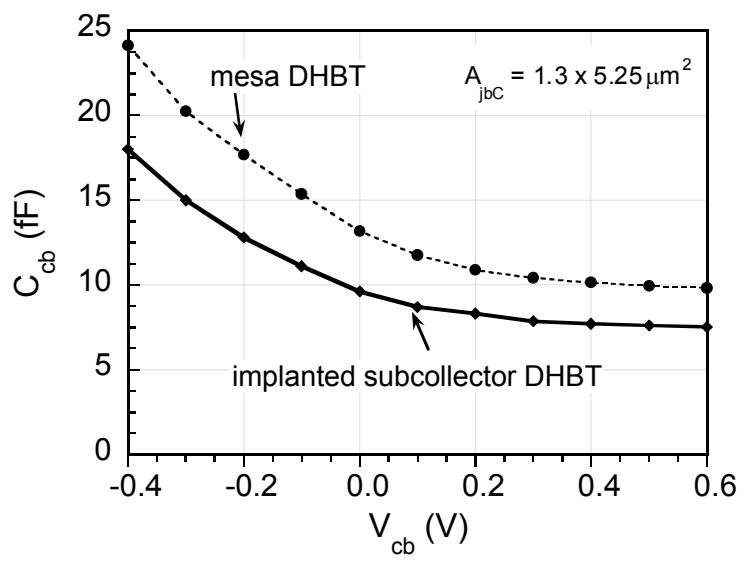

Figure 7: Capacitance Voltage characteristics

Table 1 lists the expected vs. measured reduction in $C_{c b}$ for devices of various dimensions. The slightly lower than expected reduction in capacitance is due to lithographic alignment errors and the lateral implant straggle.

\begin{tabular}{|c|c|c|c|}
\hline $\begin{array}{c}\boldsymbol{A}_{j, B C} \text { on } \\
\text { mask } \\
\left(\mu \mathbf{m}^{2}\right)\end{array}$ & $\begin{array}{c}A_{\text {pad }} \text { on } \\
\text { mask } \\
\left(\mu \mathbf{m}^{2}\right)\end{array}$ & $\begin{array}{c}\text { Effective } \\
A_{\text {pad, }} \text { isolated } \\
\left(\mu \mathbf{m}^{2}\right)\end{array}$ & $\begin{array}{c}\text { Actual (Expected) } \\
\text { reduction in } C_{c b} \\
(\mathbf{\%})\end{array}$ \\
\hline $1.1 \times 5.25$ & 2.55 & 2.07 & $25(30)$ \\
\hline $1.5 \times 5.25$ & 2.15 & 1.66 & $17(21)$ \\
\hline $1.5 \times 7.25$ & 2.75 & 2.73 & $20(20)$ \\
\hline $1.2 \times 5.25$ & 2.6 & 2.15 & $25(29)$ \\
\hline $1.2 \times 7.25$ & 2.6 & 2.43 & $21(23)$ \\
\hline $1.6 \times 7.25$ & 2.8 & 3.13 & $22(19)$ \\
\hline $2.1 \times 5.25$ & 2.85 & 2.13 & $16(17)$ \\
\hline
\end{tabular}

Table 1: Expected and measured reduction in $C_{c b}$

The hybrid pi-model extracted from RF and DC measurements is shown in Fig. 8. The thermal resistance is extracted from the Gummel curves and is $\sim 2.2 \mathrm{~K} / \mathrm{mW}$ at $J_{e}=5.5 \mathrm{~mA} / \mu \mathrm{m}^{2}$.

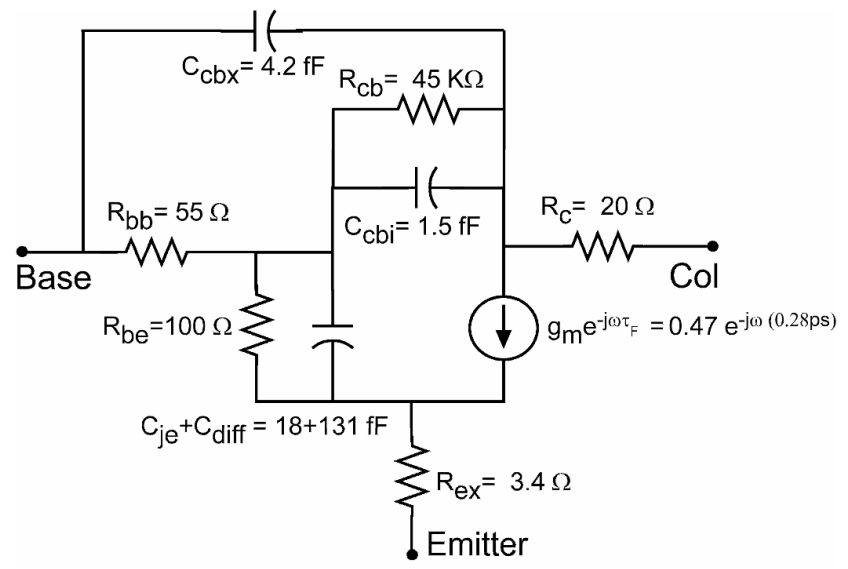

Figure 8: Hybrid- $\pi$ device model-Peak $f_{\tau}, f_{\max }$ 


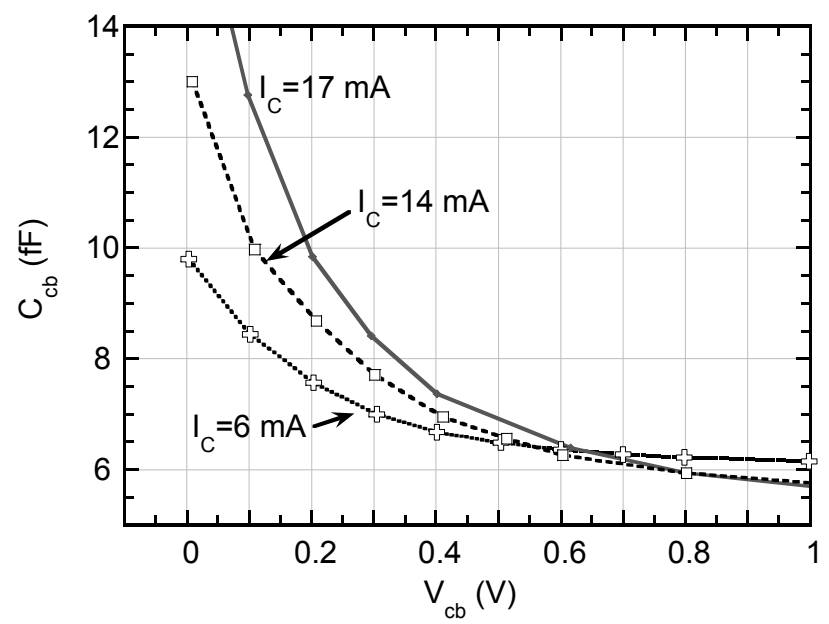

Figure 9: $C_{c b}$ variation $w /$ bias

Fig. 9 plots $C_{c b}$ as a function of both $V_{c b}$ and $I_{c}$ for the implanted subcollector DHBT. At peak $f_{\tau}, C_{c b} / I_{c} \approx 0.3 \mathrm{ps} / \mathrm{V}$. At higher current levels and low bias voltages $C_{c b}$ increases, caused by the forward biasing of the collector base junction due to the high collector resistance.

\section{Discussion}

The implanted subcollector DHBTs with Fe and Si implants, have current gain $\beta \quad 40, \eta_{c}=1.18, V_{C B O}=6.8 \mathrm{~V}, V_{C E O}=5.6 \mathrm{~V}$. The device $\beta$, ideality factors, leakage currents and breakdown voltages are consistent with those measured from the equivalent triple-mesa HBT [3]. When comparing $C_{c b}\left(V_{\mathrm{cb}}\right)$ of the two DHBTs with $120 \mathrm{~nm}$ collectors, the standard triple mesa DHBT and the implanted subcollector DHBT, a reduction in $C_{c b}$ is seen over the entire measured range of bias voltages. The interface charge is shown to be compensated by the Fe implant and the capacitance associated with the base access pad is eliminated. The hybrid- $\pi$ model indicates a high collector resistance and higher base resistance, as compared to the triple mesa DHBT [7], which are responsible for lower $f_{t}$ and $f_{\max }$. The higher base resistance is due to process and growth variations and the high collector resistance is due to insufficient annealing of the alloyed collector ohmic contacts to $N^{++}$InP. The collector ohmic contact process steps are being revised so that the collector ohmic is formed prior to base contact formation, by recess etching the base and the collector layers. Experiments on test samples indicate that a high temperature anneal $(365 \mathrm{C} / 60$ seconds) reduces the contact resistivity of the alloyed collector ohmic contact $(\mathrm{Ni} / \mathrm{GeAu} / \mathrm{Ni} / \mathrm{Au})$ to $\approx 25 \Omega \mu \mathrm{m}^{2}$. In this first demonstration of implanted subcollector DHBTs with $\mathrm{Si}$ and Fe implants, the cut-off frequencies are the best reported for implanted collector DHBTs. $C_{c b}$ due to the base access pad is eliminated and the lower $C_{c b} / I_{c}$ should enable reduced charging constants for high speed digital logic. This technology requires no additional growths, improves device planarity and is hence feasible for large scale ICs.

\section{References}

1. T. Enoki et al., "Prospects of InP-based IC technologies for 100-Gbit/sclass lightwave communications systems", International Journal of High Speed Electronics and Systems, Vol. 11, No. 1, pp. 137-158, 2001

2. M.J.W. Rodwell et al., International Journal of High Speed Electronics and Systems, "Scaling of InGaAs/InAlAs HBTs for High Speed MixedSignal and mm-Wave ICs" Vol. 11, No. 1, pp. 159-215, 2001

3. W. Hafez, J.W. Lai and M. Feng, "Submicron InP-InGaAs Single Heterojunction Bipolar Transistors With With $f_{T}$ of $377 \mathrm{GHz}$ ")", IEEE Electron Device Letters, vol. 24, no. 5, pp. 292-294, May 2003

4. H. Ishikawa, S. Miwa, T. Maruyama and M. Kamada, "Origin of n-type conduction at the the interface between epitaxial-grown layer and InP substrate and its suppression by heating in phosphine atmosphere", Journal of Applied Physics, Vol. 71(8) , pp. 3898-3903, 15 April 1992

5. Y. Dong, Z. Griffith, M. Dahlstrom, M.J.W. Rodwell, " $\mathrm{C}_{\mathrm{bc}}$ reduction in InP heterojunction bipolar transistor with selectively implanted collector pedestal", 62nd DRC Conference Digest, Vol.1, 21-23 June 2004, pp. 67-68

6. J.C. Li, M. Chen, D.A.Hitko, C.H.Fields, Binqiang Shi, R.Rajavel, P.M.Asbeck, M. Sokolich, "A submicrometer $252 \mathrm{GHz} \mathrm{f}_{\mathrm{T}}$ and $283 \mathrm{GHz}$ $\mathrm{f}_{\mathrm{MAX}}$ InP DHBT with reduced $\mathrm{C}_{\mathrm{BC}}$ using selectively implanted buried subcollector (SIBS)", IEEE Electron Device Letters, vol. 26, no. 3, pp. 136-138, March 2005

7. Z. Griffith, M.J.W. Rodwell, X-M. Fang, D. Lubyshev, Y. Wu, J.M. Fastenau, W.K. Liu, "InGaAs/InP DHBTs with $120 \mathrm{~nm}$ collector simultaneously high $\mathrm{f}_{\tau}, \mathrm{f}_{\max } \quad 450 \mathrm{GHz}$ ", IEEE Electron Device Letters, vol. 26, no. 8, pp. $530-532$, Aug. 2005 